\title{
Article \\ Structural Color Control of CoFeB-Coated Nanoporous Thin Films
}

\author{
Xiaomin Zhu ${ }^{1,+}$, Cuicui Zhao ${ }^{1,+}$, Weiwei Zhang ${ }^{1}$, Bo Zhang ${ }^{1}$, Mengtao Sun ${ }^{1}$, Xinhua Chen ${ }^{2}$, \\ Vladimir I. Belotelov ${ }^{3,4}$ and Yujun Song $1,2, * \mathbb{D}$
}

Citation: Zhu, X.; Zhao, C.; Zhang, W.; Zhang, B.; Sun, M.; Chen, X.; Belotelov, V.I.; Song, Y. Structural Color Control of CoFeB-Coated Nanoporous Thin Films. Coatings 2021, 11, 1123. https://doi.org/ 10.3390/coatings11091123

Received: 31 July 2021

Accepted: 9 September 2021

Published: 16 September 2021

Publisher's Note: MDPI stays neutral with regard to jurisdictional claims in published maps and institutional affiliations.

Copyright: (c) 2021 by the authors. Licensee MDPI, Basel, Switzerland. This article is an open access article distributed under the terms and conditions of the Creative Commons Attribution (CC BY) license (https:/ / creativecommons.org/licenses/by/ $4.0 /)$.
1 Center for Modern Physics Technology, Applied Physics Department, School of Mathematics and Physics, Beijing Engineering Research Center of Detection and Application for Weak Magnetic Field, University of Science and Technology, Beijing 100083, China; zhuxiaomin080909@163.com (X.Z.); cuicui.zhao@boip.com.cn (C.Z.); 13687641416@163.com (W.Z.); bzhang@ustb.edu.cn (B.Z.); b1603882@ustb.edu.cn (M.S.)

2 Zhejiang Key Laboratory for Pulsed Power Translational Medicine, Hangzhou Ruidi Biotechnology Company Ltd., Hangzhou 310023, China; xinhua_chen@zju.edu.cn

3 Photonic and Quantum Technologies School, Lomonosov Moscow State University, Leninskie Gori, 119991 Moscow, Russia; belotelov@physics.msu.ru

4 NTI Center for Quantum Communications, National University of Science and Technology MISiS, 119049 Moscow, Russia

* Correspondence: songyj@ustb.edu.cn

+ Contribute to this article equally.

\begin{abstract}
Unlike color dyes, structural colors only slightly fade during long-term usage. Here, structural colors were controllably achieved by constructing $\mathrm{CoFeB}$ photonic crystal layers on the surface of a nanoporous aluminum oxide (AAO) substrate by magnetron sputtering deposition. The resulting material showed a wide visible spectral response and achieved structural color control with a high resolution, high color purity, and saturation. The angle-dependent color changes of CoFeB@AAO films were further investigated by changing the incident light angle. The simulation results of the model are consistent with the experiments, which is significant in practical applications. This strategy may have great potential applications for solid structure color coatings, anti-counterfeiting and security, information storage, and electromagnetic sensors.
\end{abstract}

Keywords: anodic aluminum oxide (AAO); $\mathrm{CoFeB}$; magnetron sputtering deposition; thin film; structural color

\section{Introduction}

In contrast to coloration using dyes, structural colors are usually produced by geometric structures. Periodic micro/nanostructure arrays often display intrinsic structural colors due to their ability to control the transmission of light. Structural colors are efficient in terms of energy consumption and light utilization, representing the most vivid and brightest colors in nature, and showing little fading during long-term applications [1,2]. In nature, structural colors are widely observed in flowers, insects, butterflies, worms, fish [3], and chameleons $[4,5]$.

Photonic crystals are micro/nanostructures formed by dielectric periodic arrays with different refractive indices. Photonic crystals also display structural colors due to their periodic arrangement, Bragg diffraction, interference [6], and scattering of light propagating on the surface and inside of the photonic crystals [7]. These properties of photonic crystals allow the material to selectively absorb light of a specific frequency and show different colors [8]. Due to its full-color emission and stable optical signal, structural color has unique application in anti-counterfeiting [9], optical display devices [10], and electromagnetic sensors [11]. 
In principle, optical signals are mainly adjusted by three strategies. The first is to control the transmission and reflection of visible light. The second is to change the refractive index and the incident angle. The third is to manipulate the nanostructures of photonic crystals. One of the most efficient methods for structural colors is to fabricate periodic arrayed artificial photonic crystals on a substrate, which can be a functional polymer such as a hydrogel, polyelectrolyte, or elastomers [12,13]. However, preparation of these complicated structures is very difficult, and an external field is often required to improve the desired saturation of colors [14]. An alternative is to directly use field-sensitive materials such as magnetic alloys to construct photonic crystals $[8,15]$. Photonic crystals can be constructed by magnetron sputtering deposition [16], atomic layer deposition (ALD) [8], etc. Photonic crystals are stable and easy to be prepared, which provides favorable conditions for future application. $\mathrm{CoFeB}$ is a strong magnetic material, which has excellent performance in the construction of vertical magnetic anisotropic films [16-18], magnetic tunnel junctions, and other structures [19]. And AAO templates have been developed since 1953 [20]. AAO template assisted fabrication process has become a mature, stable, and efficient way to construct photonic crystals, which has good performance in the construction of a variety of photonic crystals [21-26].

In this study, $\mathrm{CoFeB@AAO} \mathrm{photonic} \mathrm{crystals} \mathrm{were} \mathrm{constructed} \mathrm{by} \mathrm{directly} \mathrm{coating} \mathrm{a}$ layer of magnetic $\mathrm{Co}_{40} \mathrm{Fe}_{40} \mathrm{~B}_{20}$ alloy on the $\mathrm{AAO}$ template. The resulting photonic crystal nanostructures were manipulated by adjusting the thickness of the CoFeB layers and the pore diameter of the AAO template. The thickness of the CoFeB layer is controlled by adjusting the magnetron sputtering time, and the aperture is adjusted by changing different AAO substrates. The angle dependence was further studied by changing the incident light angle. The obtained structural colors could be controlled in the visible region ( $\lambda: 350-800 \mathrm{~nm}$ ). Finally, we modulated the three primary colors (red, green, and blue) and other middle colors. Our results suggest that a wide range of chromatographic responses can be achieved in the visible region. Color generation was analyzed by studying the nanostructure, reflectivity, and interference coupling behavior. Simultaneously, the related physical mechanism was further studied by theoretical simulations based on the structure of the self-assembled photonic crystals, which matched the experimental results very well.

\section{Experimental Section}

\subsection{Sample Preparation}

Substrate-confined AAO templates can be prepared by a two-step anodic oxidation process $[17,18]$. However, for the convenience of the experiment, we directly purchased it from Shanghai Shangmu Technology Co., Ltd. (Shanghai, China). Pure ferromagnetic $\mathrm{Co}_{40} \mathrm{Fe}_{40} \mathrm{~B}_{20}$ thin films with a thickness of $16.3 \mathrm{~nm}$ were deposited on AAO templates with pore diameters of 30,50,70, and $90 \mathrm{~nm}$ (AAO interpore distance: $110 \mathrm{~nm}$; pore depth: $300 \mathrm{~nm}$ ) as sample series 1-1. After this, we selected AAO substrates with $90 \mathrm{~nm}$ pore diameter and deposited $\mathrm{Co}_{40} \mathrm{Fe}_{40} \mathrm{~B}_{20}$ films with thicknesses of 16.3, 32.7, and $49.0 \mathrm{~nm}$ (sputtering duration: 3, 6, and $9 \mathrm{~min}$, respectively) as sample series 1-2.

In sample series 2, the structural parameters of the CoFeB@AAO film were fixed (the aperture of $\mathrm{AAO}$ was $90 \mathrm{~nm}$ with sputtering duration $3 \mathrm{~min}$ ), and we chose different incident light angles to explore whether different incident light angles would affect the structural color.

All samples were deposited at room temperature by magnetron sputtering deposition at a base pressure of $1.8 \times 10^{-4} \mathrm{~Pa}[17,19]$. During this process, the Ar gas pressure in the magnetron sputtering chamber was controlled at $0.75 \mathrm{mTorr}$, and the power density was $5.1 \mathrm{~W} / \mathrm{cm}^{2}$. Sputtering angle was fixed at $45^{\circ}$. 


\subsection{Characterization of the Morphology and Composition of CoFeB@AAO Films}

The surface and cross-section morphologies and the position analysis of the CoFeB@AAO films were characterized using field emission scanning electron microscopy (FE-SEM, SUPRA 55, Zeiss, Jena, Germany), which was coupled with energy-dispersive X-ray spectroscopy (EDS). The thickness of the CoFeB was measured by a step profiler (Bruker Countor GT K, Bruker Daltonic Inc., Billerica, MA, USA). When the sample thickness was measured, the sample thickness was not directly measured on the photonic crystal because it was difficult to find the boundary between $\mathrm{CoFeB}$ and $\mathrm{AAO}$ after the sample is completed. Therefore, we chose to measure the sputtering rate of $\mathrm{CoFeB}$ on the smooth film and control the sample thickness by controlling the time. Therefore, the actual thickness had a deviation of about $2 \mathrm{~nm}$. We purchased CoFeB target material from Beijing Zhongnuo New Material Co., LTD. (Beijing, China).

From Figure 1, the structure of the AAO template presented a uniform hexagonal honeycomb arrangement of nanopores on the overview (a) image and (b) cross-section, which were further verified by their SEM images (Figure 2). The CoFeB coating the AAO thin films could be regarded as a Bragg reflector, following the Bragg equation below in Equation (1) [20].

$$
\sum 2 n_{i} d_{i} \cos \theta_{i}=m \lambda
$$

where $n_{i}$ represents the effective refractive index, $d_{i}$ represents the thickness, $\theta_{i}$ represents the incident angle of the $i$ th layer, $m$ is the diffraction grades, and $\lambda$ is the wavelength. Equation (1) indicates that the AAO template should be colorless in the visible range.

(a)

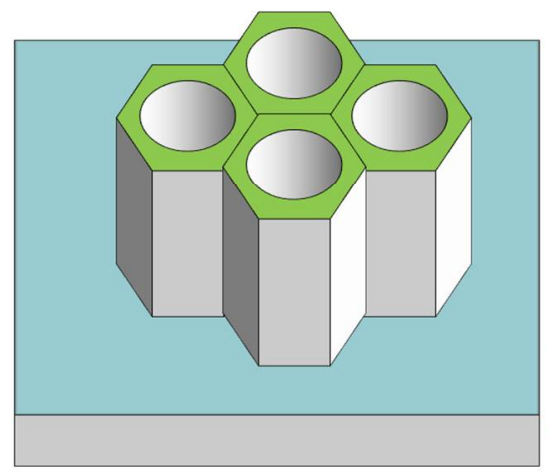

(b)

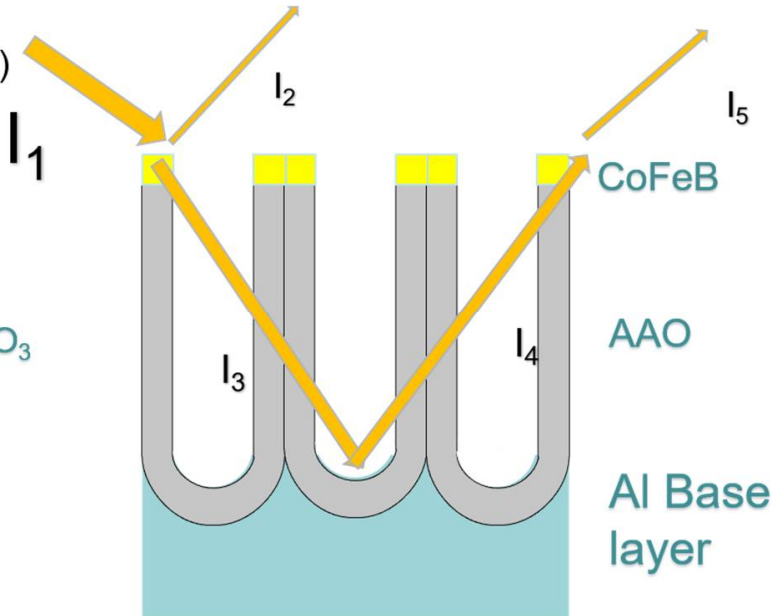

Figure 1. (a) Simplified 3D model of the AAO/Al templates. (b) Interferometric equivalent optical path diagrams of the $\mathrm{AAO} / \mathrm{Al}-$ based $\mathrm{CoFeB}$ nanostructure arrays.

The equivalent optical path of the AAO template with a $45^{\circ}$ incident angle is described in detail in Figure 1. It can be seen from Figure 1 that once the incident light $I_{1}$ hits the air-AAO interface, a small part of light $I_{2}$ will be reflected into the air. Most of the light $I_{3}$ passes through AAO and then reflects from the AAO layer to become the reflecting light $I_{4} . I_{4}$ passes through the CoFeB film and is reflected as $I_{5}$. Through the reflection and absorption of the CoFeB layer, the intensity of $I_{2}$ and $I_{5}$ can be adjusted to make the intensity of the two beams approximately equal. There is a fixed phase difference between them, which matches the interference conditions and produces bright colors. 

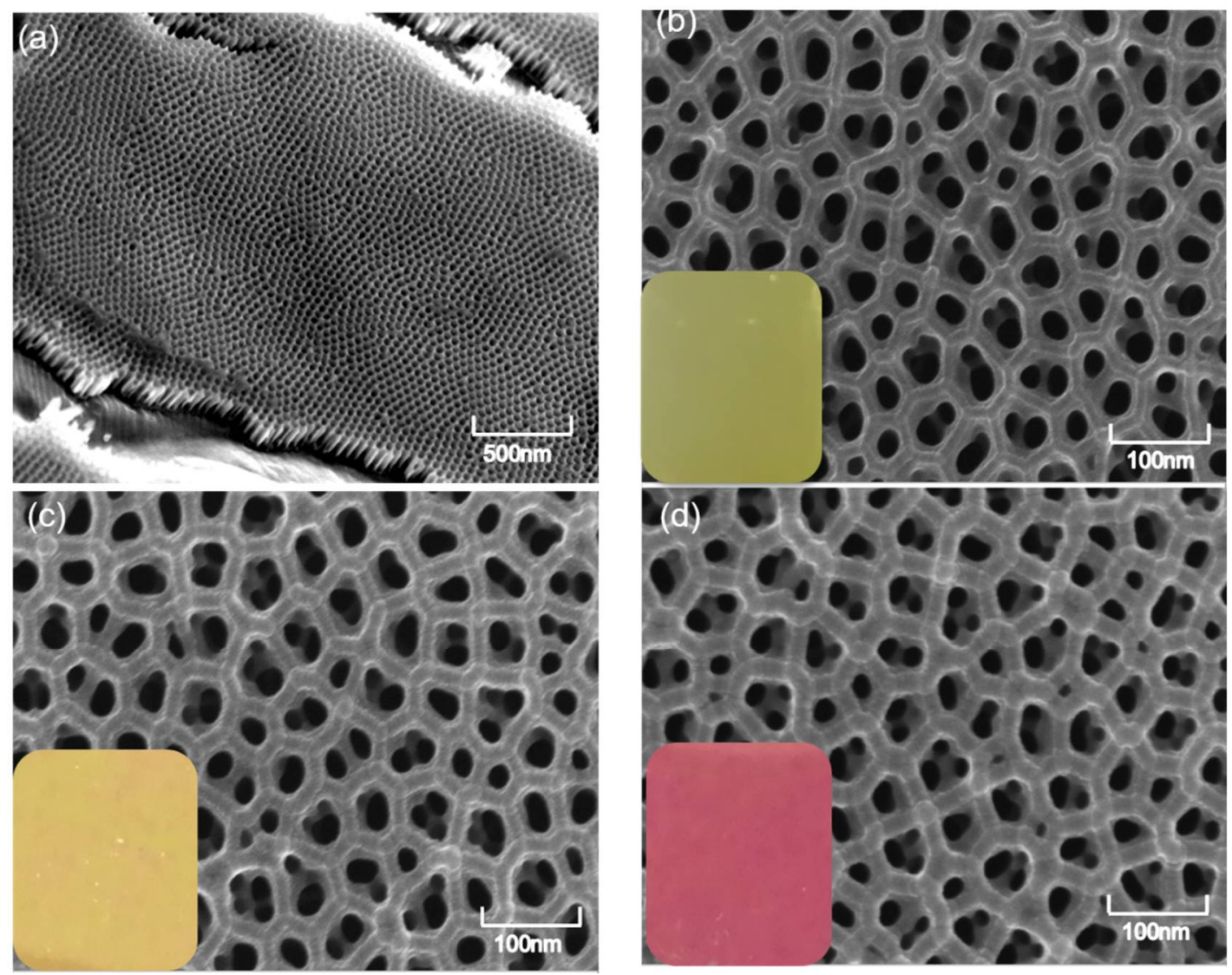

Figure 2. Structural colors of a representative sample of a CoFeB@AAO thin film with a pore diameter of $90 \mathrm{~nm}$. Its top surface has a petal-like structure, forming a hexagon-like structure whose side lengths are equal to the pore spacing (110 $\mathrm{nm}$ ) and a hole depth of $300 \mathrm{~nm}$. (a) SEM image of the AAO, (b) film thickness $=16.3 \mathrm{~nm}$, (c) film thickness $=32.7 \mathrm{~nm}$, and (d) the cross-sectional image of the $49.0 \mathrm{~nm}$ film.

After series 1-1, 1-2, and 2 have been tested, their reflectance can be obtained. Because our samples were in the visible region, we chose the direct selection method to calculate their tristimulus values $(X, Y, Z)$. Using the standard method recommended by Commission Internationale de L'Eclairage (CIE), $X, Y$, and $Z$ are given by Equation (2).

$$
X=k \sum_{i=0}^{n} S\left(\lambda_{i}\right) \bar{x}\left(\lambda_{i}\right) R\left(\lambda_{i}\right) \Delta \lambda Y=k \sum_{i=0}^{n} S\left(\lambda_{i}\right) \bar{y}\left(\lambda_{i}\right) R\left(\lambda_{i}\right) \Delta \lambda Z=k \sum_{i=0}^{n} S\left(\lambda_{i}\right) \bar{z}\left(\lambda_{i}\right) R\left(\lambda_{i}\right) \Delta \lambda
$$

where $\Delta \lambda=1, k=100 / \sum S\left(\lambda_{J}\right) y\left(\lambda_{j}\right)(J=0,1 \ldots \ldots . . n), S\left(\lambda_{i}\right)$ : Source energy distribution, $X(\lambda), Y(\lambda)$, and $Z(\lambda)$ : CIE 1964 Standard observer tristimulus value curve.

After obtaining the $X, Y$, and $Z$ values, the color of series 1-1, 1-2, and 2 can be calculated by transformation into red, green, and blue (RGB) values by Formula (3):

$$
\begin{aligned}
& {[X]=\left[\begin{array}{lll}
0.433953 & 0.376219 & 0.189828
\end{array}\right][R]} \\
& {[Y]=\left[\begin{array}{lll}
0.212671 & 0.715160 & 0.072169
\end{array}\right][\mathrm{G}]} \\
& {[Z]=\left[\begin{array}{lll}
0.017758 & 0.109477 & 0.872765
\end{array}\right][B]}
\end{aligned}
$$

To verify our experimental scheme, the corresponding model was built, and the reflectivity curves of series 1-1, 1-2, and 2 were simulated under the corresponding conditions and compared with the actual values.

\section{Results and Discussion}

CoFeB@AAO films were prepared by magnetron sputtering on the surface of an AAO template. Figure 2 shows a representative SEM image of a CoFeB@AAO film with a pore 
diameter of $90 \mathrm{~nm}$. The top surface of the film has a petal-like structure consisting of six connected nanoparticles that form an equilateral triangle with the sides equal to the pore spacing $(110 \mathrm{~nm})$. Figure $2 a$ shows the empty SEM images of an AAO template. Figure $2 b$ presents the SEM micrograph of the CoFeB@AAO film prepared with a sputtering time of $3 \mathrm{~min}(16.3 \mathrm{~nm})$. As can be seen from Figure $2 b$, a CoFeB layer was evenly distributed on the surface of the AAO template. The bottom left corner shows the real color of the structural thin film after deposition, a bright grass-green color. Figure 2c shows the CoFeB@AAO film prepared with a sputtering time of $6 \mathrm{~min}(32.7 \mathrm{~nm})$. The structural color of this film after deposition changed from grass-green to bright yellow. Figure 2d presents the CoFeB@AAO film prepared at a sputtering time of $9 \mathrm{~min}(49.0 \mathrm{~nm})$. The thickness of the CoFeB layer increased significantly and was thicker than that of the CoFeB layer in Figure 2b,c, whose structural color changed from bright yellow to red.

In sample series 1-1, the pores of the AAO template were adjusted using a $\mathrm{CoFeB}$ layer with a sputtering time of $t=3 \mathrm{~min}$ at an incident light angle of $45^{\circ}$. Figure $3 \mathrm{a}$ shows the reflectivity and corresponding colors of sample series 1-1. The curves are the reflectivity spectra of CoFeB@AAO films, whose corresponding structural colors are inserted in the bottom right corners. By observing the reflectivity of the samples with different pore sizes, it was found that their peaks and valleys were obviously different. As can be seen from Formula (1), we changed the pores of the AAO template, resulting in different interfered wavelengths, which also resulted in the appearance of different colors on the sample. At the same time, we also directly calculated the tristimulus values of $\mathrm{X}, \mathrm{Y}$, and $\mathrm{Z}$ to obtain the corresponding colors from the reflectivity. Figure 3a shows that the structural color of CoFeB@Al (the control group) appeared red-brown, but as the diameter of the AAO template changed from $30 \mathrm{~nm}$ to $90 \mathrm{~nm}$, the structural colors changed significantly. In sample series 1-2, the thickness of the CoFeB@AAO film with a pore diameter of $90 \mathrm{~nm}$ increased from $60 \mathrm{~nm}$ to $120 \mathrm{~nm}$, whose reflectivities and corresponding colors are shown in Figure $3 b$. As can be seen from Figure $3 b$, as the thickness of the film is increased, the peaks and the valleys of the reflectivity curves red-shift, leading to a change in the structural colors from grass-green to red.

As shown in Figure 3c, when the thickness of $\mathrm{CoFeB}$ and the pore diameter of the AAO template were the same, series 2 samples showed color changes at different incident angles compared with the pure $\mathrm{CoFeB}$ on the $\mathrm{Al}$ substrate (control). When the incident light angle changed, the color of the CoFeB@AAO film red-shifted and became yellow.

In addition, as the incident light angle changed, the brightness of the structural color changed. When a high reflection and low absorption simultaneously occur in the visible region, the amplitude ratio of the reflection to the absorption will be dramatically increased, leading to distinct color saturation. Thus, as the incident angle was increased, the reflection and absorption peaks broadened, and the corresponding colors appeared more vivid.

Figure $3 \mathrm{~d}$ gives the reflectivity of thin films when the thickness was increased from $40 \mathrm{~nm}$ to $120 \mathrm{~nm}$, using the pure AAO template as a control. When the thickness was increased to a certain value, the amplitudes between the reflection and absorption peaks tended to decrease, even though the ridges and valley of the reflectivity peaks underwent a red-shift. As can be seen from Figure 1, when the thickness of CoFeB gradually increases, more and more parts of light are absorbed. When high reflection and low absorption in the visible area occur at the same time, a clear color will be produced. Therefore, when the thickness increases to a certain critical value, the color will dim gradually. 

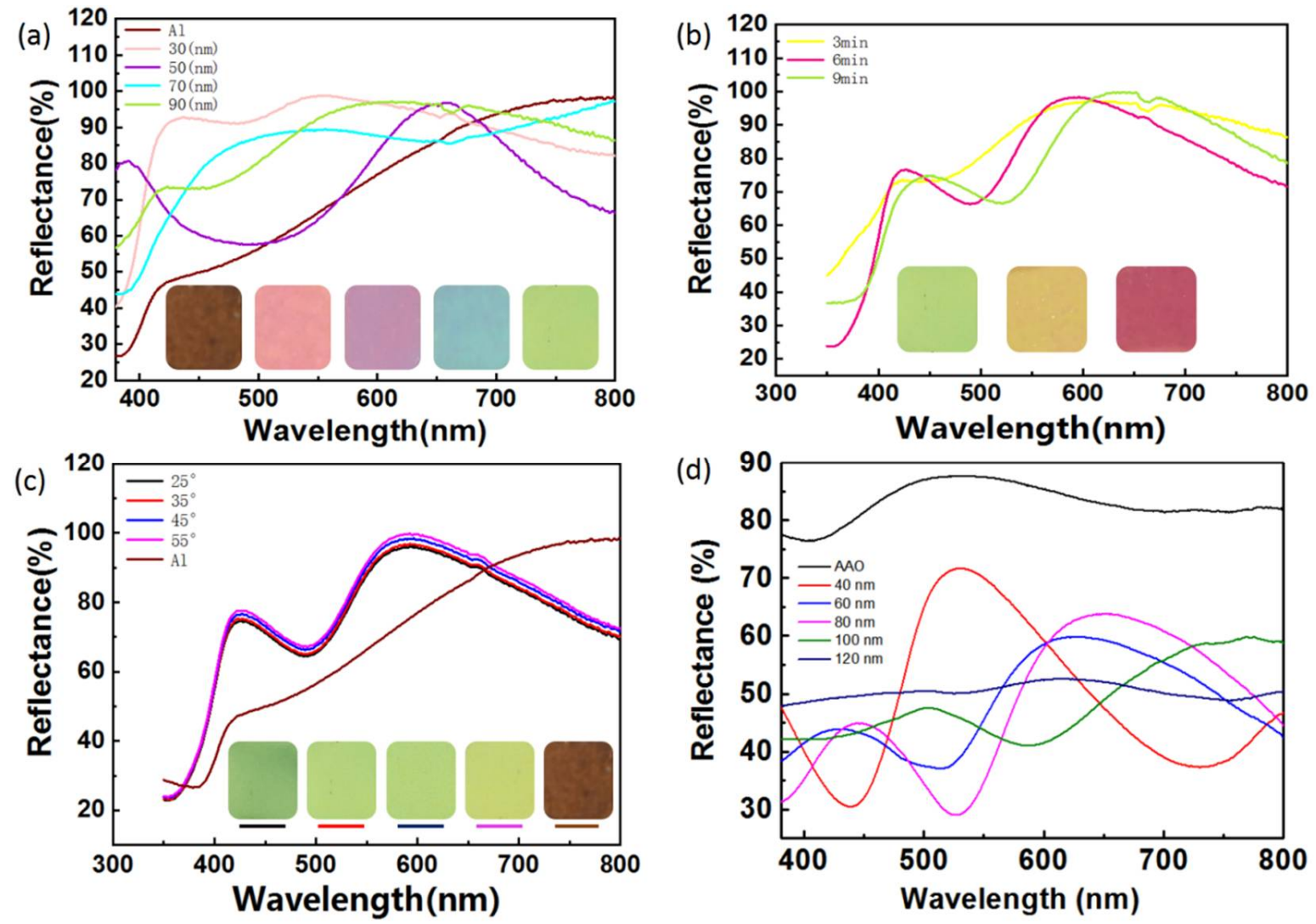

Figure 3. Reflectivity and structural colors of sample series 1 and 2. (a) Reflectivity and structural color of sample series 1-1. (b) Reflectivity and structural color of sample series 1-2. (c) The induced light angle-dependent reflectivity of sample series 2.

(d) The thickness-dependent reflectivity of sample series 1-2.

In addition, since the AAO template is a relatively uniform nanoporous template, the film deposited on it should have a relatively uniform honeycomb structure. We used the finite element method to establish the corresponding model structure (Figure 4) according to the structure in Figure 1. The reflectivities of the corresponding CoFeB@AAO films with different thicknesses were then calculated, as shown in Figure 5.
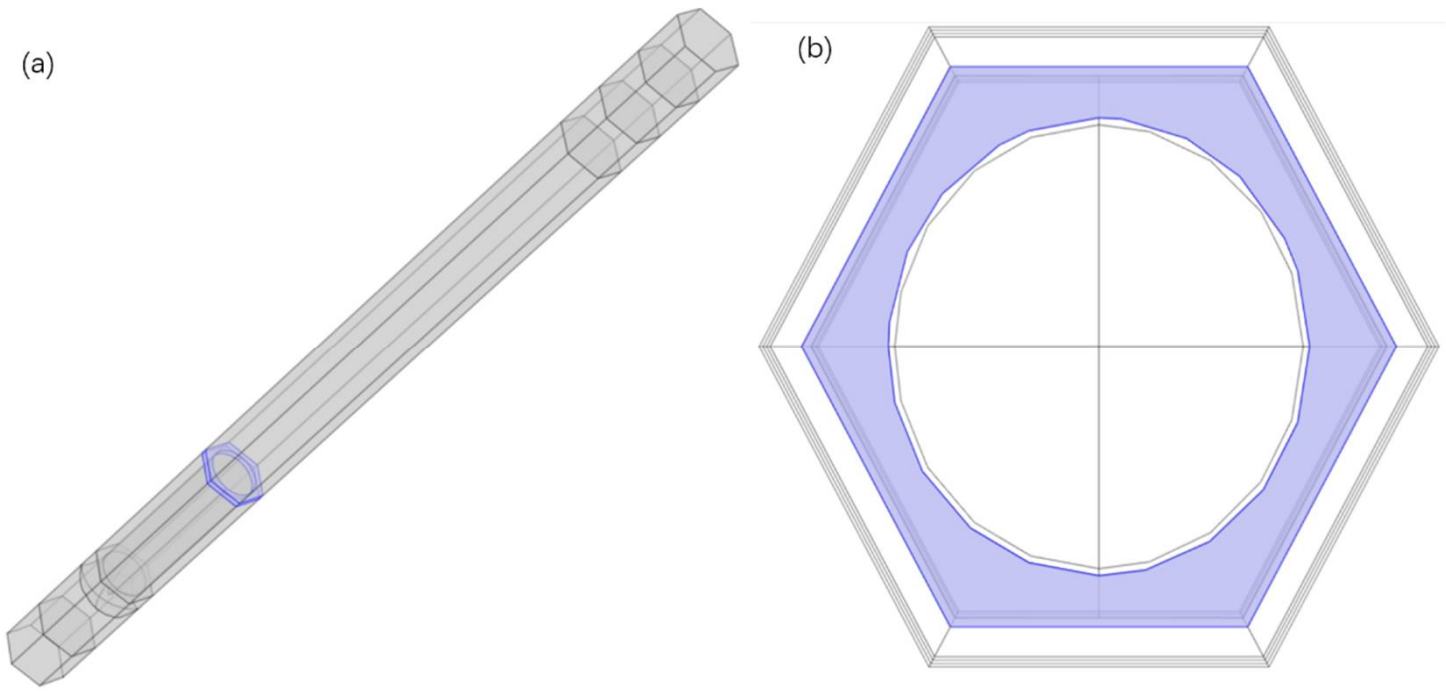

Figure 4. Periodic CoFeB@AAO thin film model diagrams: (a) 3D model diagram and (b) XOY plane diagram. The blue part of the figure is the $\mathrm{CoFeB}$ layer. 
(a)

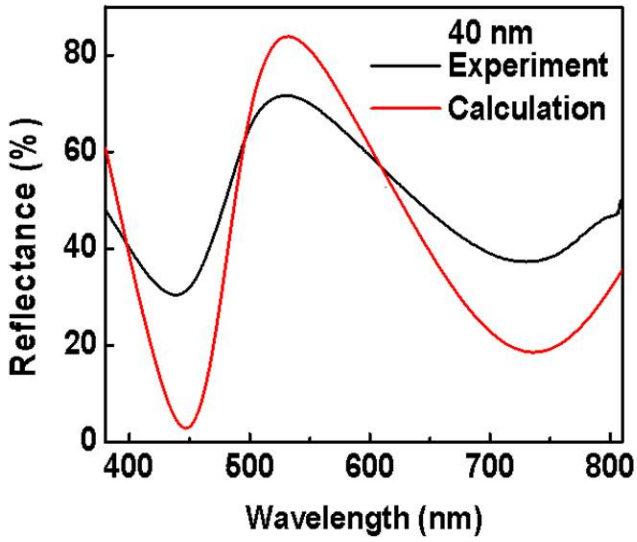

(c)

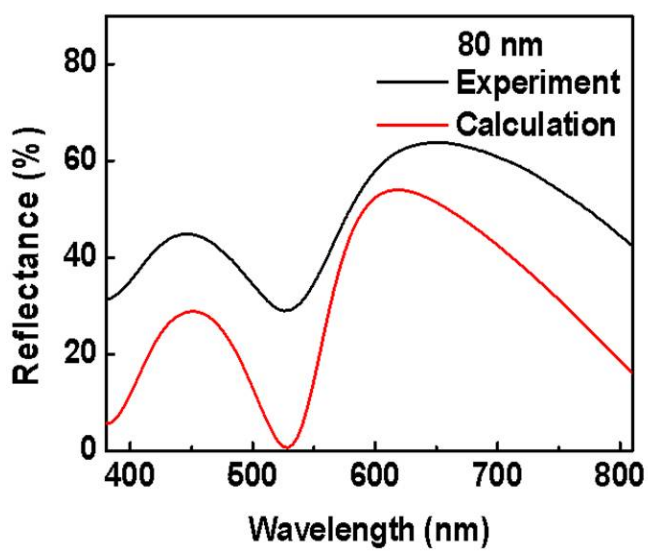

(b)

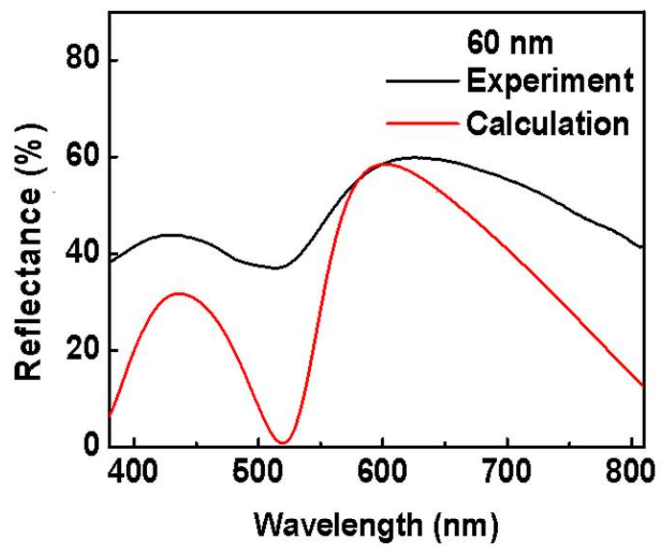

(d)

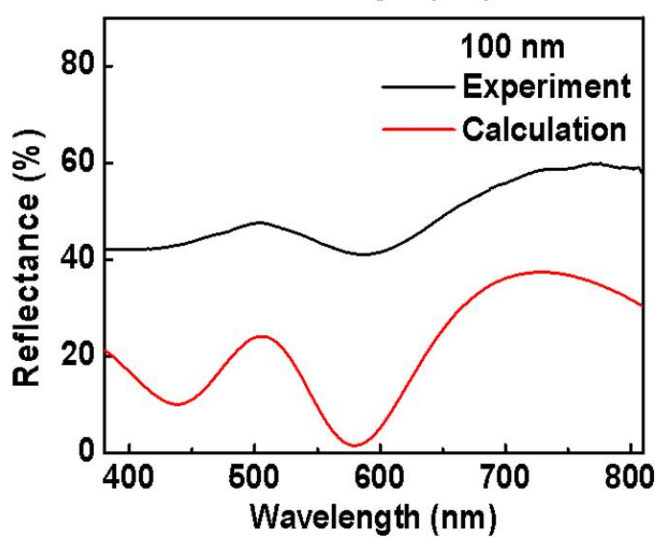

Figure 5. Calculated and experimental reflectance spectra of CoFeB@AAO films with controlled coating thicknesses on AAO with a $90 \mathrm{~nm}$ pore size: (a) $40 \mathrm{~nm}$; (b) $60 \mathrm{~nm}$; (c) $80 \mathrm{~nm}$; (d) $100 \mathrm{~nm}$.

Figure $4 \mathrm{a}$ exhibits a 3D image of the model, whereas Figure $4 \mathrm{~b}$ is a periodic image in the XOY plane that periodically extends outward from the center of the hole. Figure 5 shows the actual reflectivity and calculated values when the CoFeB thickness was $40,60,80$, and $100 \mathrm{~nm}$, respectively. The reflectivity variations obtained from the established model and experiment results have ridges and valleys nearly in the same positions. The deviation in the intensities occurred due to variations between the models established using a much more uniform structure and the actual AAO templates. It can be shown in Figure 2, suggesting that the pore structures are not strictly uniform, which attributes to some variations between the simulation and the experiment.

\section{Conclusions}

Here, we presented a structural color control method by coating a CoFeB layer on an AAO photonic crystal thin film via magnetron sputtering deposition. The obtained films displayed high resolution, high color purity, and saturation in the visible region. The photonic crystal nanostructure was manipulated by adjusting the thickness of the CoFeB films and the diameter of the AAO template. In the visible region, the film displayed nearly perfect light absorption by simply adjusting the structure of the photonic crystal substrate. This method shows great potential in vivid and stable structure color coatings, anti-counterfeiting marks, optical information storage, and electromagnetic sensors.

Author Contributions: Conceptualization, X.Z. and C.Z.; methodology, W.Z.; software, X.Z. and W.Z.; validation, Y.S. and M.S.; formal analysis, B.Z.; investigation, X.C.; resources, V.I.B.; data curation, X.Z.; writing-original draft preparation, X.Z., C.Z.; writing-review and editing, X.Z.; visualization, W.Z.; supervision, Y.S.; project administration, Y.S.; funding acquisition, Y.S. and V.I.B. All authors have read and agreed to the published version of the manuscript. 
Funding: This study was financially supported by BRICS STI Framework Programme by NSFC (No. 51861145309), the National Natural Science Foundation of China (No. 51971029), the National S\&T Major Project of China (No. 2018ZX10301201), Development of ultrasensitive magneto-optical and electrical biosensors (contract No.: 2019-0649) from Hangzhou Ruidi Biotechnology Company Ltd., Russian Foundation for Basic Research (projects No 18-52-80038 and 18-29-20113), China Postdoctoral Science Foundation (No. 2020M680336) and Teaching Reform Project (jgzd 20201006).

Institutional Review Board Statement: Not applicable.

Informed Consent Statement: Not applicable.

Data Availability Statement: Not applicable.

Acknowledgments: Authors are grated of the support school of Mathematics and Physics, University of Science and Technology Beijing, the interdisciplinary "Scientific and Educational School of Moscow University Photonic and Quantum technologies, Digital medicine, Shanghai Shangmu Technology Co., Ltd. (Shanghai, China), and China Scientific Compass Data Measurement Platform. Authors also appreciate the constructive comments in the revision of this article.

Conflicts of Interest: There is no interest conflict among authors.

\section{References}

1. Zhao, Y.; Xie, Z.; Gu, H.; Zhu, C.; Gu, Z. Bio-Inspired Variable Structural Color Materials. Chem. Soc. Rev. 2012, 41, 3297-3317. [CrossRef] [PubMed]

2. Shang, L.; Zhang, W.; Ke, X.; Zhao, Y. Bio-Inspired Intelligent Structural Color Materials. Mater. Horiz. 2019, 6, 945-958. [CrossRef]

3. Lythgoe, J.N.; Shand, J. The Structural Basis for Iridescent Colour Changes in Dermal and Corneal Irddophores in Fish. J. Exp. Biol. 1989, 141, 313-325. [CrossRef]

4. Gao, X.; Xin, Y.; Xi, Y.; Liang, X.; Lei, J. The Dry-Style Antifogging Properties of Mosquito Compound Eyes and Artificial Analogues Prepared by Soft Lithography. Adv. Mater. 2010, 19, 2213-2217. [CrossRef]

5. $\quad$ Parker, A.R.; Mcphedran, R.C.; Mckenzie, D.R.; Botten, L.C.; Nicorovici, N. Aphrodite's Iridescence. Nature 2001, 409, 36-37. [CrossRef]

6. Sato, O.; Kubo, S.; Gu, Z.-Z. Structural Color Films with Lotus Effects, Superhydrophilicity, and Tunable Stop-Bands. Acc. Chem. Res. 2009, 42, 1-10. [CrossRef] [PubMed]

7. Jang, J.; Badloe, T.; Yang, Y.; Lee, T.; Rho, J. Spectral Modulation through the Hybridization of Mie-Scatterers and Quasi-Guided Mode Resonances: Realizing Full and Gradients of Structural Color. ACS Nano 2020, 14, 15317-15326. [CrossRef] [PubMed]

8. Lee, K.; Kim, H.; Kim, J.H.; Choi, D. Structural Color and near-Infrared Tunability of Ruthenium-Coated Anodic Aluminum Oxide by Atomic Layer Deposition. Scr. Mater. 2020, 187, 125-129. [CrossRef]

9. Zhao, Z.; Wang, H.; Shang, L.; Yu, Y.; Fu, F.; Zhao, Y.; Gu, Z. Bioinspired Heterogeneous Structural Color Stripes from Capillaries. Adv. Mater. 2017, 29, 1704569. [CrossRef] [PubMed]

10. Zhao, Y.; Zhao, Y.; Hu, S.; Lv, J.; Ying, Y.; Gervinskas, G.; Si, G. Artificial Structural Color Pixels: A Review. Materials 2017, $10,944$. [CrossRef]

11. Park, T.H.; Yu, S.; Cho, S.H.; Kang, S.H.; Kim, Y.; Kim, M.J.; Eoh, H.; Park, C.; Jeong, B.; Lee, S.W.; et al. Block Copolymer Structural Color Strain Sensor. NPG Asia Mater. 2018, 10, 328-339. [CrossRef]

12. Lee, H.S.; Shim, T.S.; Hwang, H.; Yang, S.M.; Kim, S.H. Colloidal Photonic Crystals toward Structural Color Palettes for Security Materials. Chem. Mater. 2013, 25, 2684-2690. [CrossRef]

13. Yang, Z.; Chen, G.; Huang, Y.; Liang, Q.; Shen, H. Development of Bright and Low Angle Dependent Structural Color Coatings Via Electrophoretic Deposition with Variable Voltage. Surf. Interfaces 2021, 24, 101045. [CrossRef]

14. Gu, Z.-Z.; Fujishima, A.; Sato, O. Fabrication of High-Quality Opal Films with Controllable Thickness. Chem. Mater. 2002, 14, 760-765. [CrossRef]

15. Zhu, C.; Xu, W.; Chen, L.; Zhang, W.; Xu, H.; Gu, Z.-Z. Magnetochromatic Microcapsule Arrays for Displays. Adv. Funct. Mater. 2011, 21, 2043-2048. [CrossRef]

16. Cheng, F.; Gao, J.; Stan, L.; Rosenmann, D.; Czaplewski, D.; Yang, X. Aluminum Plasmonic Metamaterials for Structural Color Printing. Opt. Express 2015, 23, 14552-14560. [CrossRef]

17. Yi, L.; Tao, Y.; Zhengyong, Z.; Huicai, Z.; Khamis Masoud, K.; Kaigui, Z. High thermal stability in W/MgO/CoFeB/W/CoFeB/W stacks via ultrathin $\mathrm{W}$ insertion with perpendicular magnetic anisotropy. J. Magn. Magn. Mater. 2016, 410, $123-127$.

18. Xiong, D.; Peng, S.; Lu, J.; Li, W.; Wu, H.; Li, Z.; Cheng, H.; Wang, Y.; Back, C.H.; Wang, K.L.; et al. Modulation of thermal stability and spin-orbit torque in IrMn/CoFeB/MgO structures through atom thick W insertion. Appl. Phys. Lett. 2020, 117, 212401. [CrossRef]

19. Zhou, Z.; Marcon, P.; Devaux, X.; Pigeat, P.; Bouché, A.; Migot, S.; Jaafar, A.; Arras, R.; Vergnat, M.; Ren, L.; et al. Large Perpendicular Magnetic Anisotropy in $\mathrm{Ta} / \mathrm{CoFeB} / \mathrm{MgO}$ on Full-Coverage Monolayer MoS2 and First-Principles Study of Its Electronic Structure. arXiv 2021, arXiv:2106.10317. 
20. Isogami, S.; Shiokawa, Y.; Tsumita, A.; Komura, E.; Ishitani, Y.; Hamanaka, K.; Taniguchi, T.; Mitani, S.; Sasaki, T.; Hayashi, M. Spin-orbit torque driven magnetization switching in $\mathrm{W} / \mathrm{CoFeB} / \mathrm{MgO}$-based type-Y three terminal magnetic tunnel junctions. Sci. Rep. 2021, 11, 16676. [CrossRef]

21. Keller, F.; Hunter, M.S.; Robinson, D.L. Structural Features of Oxide Coatings on Aluminum. J. Electrochem. Soc. 1953, $100,411$. [CrossRef]

22. Li, D.; Wu, A.; Wan, Q.; Li, Z. Controllable fabrication of polymeric nanowires by NIL technique and self-assembled AAO template for SERS application. Sci. Rep. 2021, 11, 14929. [CrossRef]

23. Song, Y.; Wang, Y.; Li, B.B.; Fernandes, C.; Ruda, H.E. Interface Interaction Induced Ultra-Dense Nanoparticles Assemblies. Nanoscale 2013, 5, 6779-6789. [CrossRef] [PubMed]

24. Song, Y.; Yin, W.; Fernandes, C.; Ruda, H.E. Fabrication of One-Dimension Znse and Zno Nanostructures Via Anodic Alumina Template Assisted Vapor-Liquid-Solid Growth Process. Thin Solid Film. 2013, 548, 130-137. [CrossRef]

25. Song, Y.; Yin, W.; Wang, Y.H.; Zhang, J.P.; Yan, W.; Wang, R.; Han, J.; Wu, W.; Nair, S.V.; Ruda, H.E. Magneto-Plasmons in Periodic Nanoporous Structures. Sci. Rep. 2014, 4, 4991. [CrossRef]

26. Wang, X.; Zhang, D.; Zhang, H.; Ma, Y.; Jiang, J.Z. Tuning Color by Pore Depth of Metal-Coated Porous Alumina. Nanotechnology 2011, 22, 305306. [CrossRef] 